\title{
Spatial negative priming: In touch, it's all about location
}

\author{
Ann-Katrin Wesslein ${ }^{1,2} \cdot$ Charles Spence $^{2} \cdot$ Frank Mast $^{1,2} \cdot$ Christian Frings $^{1}$
}

Published online: 20 November 2015

(C) The Psychonomic Society, Inc. 2015

\begin{abstract}
Spatial negative priming (SNP) refers to the finding that responses to stimuli that are presented from previously ignored locations are slowed relative to responses to stimuli presented from previously unstimulated locations. To date, this effect has been demonstrated in vision, audition, and touch. Importantly, however, the cognitive processes involved differ between vision and audition. Although SNP is attributable to feature mismatch in the auditory modality, it is primarily caused by response inhibition in vision. To date, the locus of SNP in touch has not been established, though recently it has been shown that tactile SNP is not modulated by feature mismatch. Here, we demonstrate that in touch, as compared to vision and audition, SNP is more sensitive to the location features - and not solely to the response and stimulus features. Thus, in stark contrast to identity-based negative priming, in which responses to previously ignored stimuli (based on the stimulus identity) can be explained by the same mechanisms in all three sensory modalities, SNP would appear to be caused by different processes in each sensory modality, thus suggesting that the processing of the spatial properties of distractors is modality-specific.
\end{abstract}

Keywords Touch $\cdot$ Spatial attention $\cdot$ Spatial negative priming $\cdot$ Response inhibition

Ann-Katrin Wesslein

wesslein@uni-trier.de

1 Department of Psychology, University of Trier, Trier, Germany

2 Crossmodal Research Laboratory, Department of Experimental Psychology, University of Oxford, Oxford, UK
Responses to a stimulus (target) that has recently been ignored (distractor) are often slower and more error prone than responses to targets that have not been presented previously (see Fox, 1995; Frings, Schneider, \& Fox, 2015; Tipper, 2001, for reviews). This phenomenon, labelled negative priming (NP; Tipper, 1985), has generally been investigated by presenting target and distractor stimuli (as defined by a given feature - e.g., color), with the response being based on the identity of the target stimulus. The NP paradigm includes two displays, one called the prime display and the other the probe display. The NP effect is computed as the performance difference between those trials on which the probe target and probe distractor are unrelated to the stimuli presented during the prime display (control trials) and those trials on which the prime distractor serves as the target during the probe display (ignored repetition trials).

Since NP was originally reported nearly half a century ago (Dalrymple-Alford \& Budayr, 1966), several approaches to explaining NP have been put forward in the literature. Traditionally, inhibition accounts (e.g., Houghton \& Tipper, 1994; Tipper, 1985) can be differentiated from retrieval-based accounts (e.g., Milliken, Joordens, Merikle, \& Seiffert, 1998; Neill, Valdes, Terry, \& Gorfein, 1992). According to inhibition theories, NP is thought to reflect an aftereffect of the active inhibition of the prime distractor that becomes evident on the probe display. By contrast, according to retrieval-based theories, ignored distractors are associated with a kind of "donot-respond" tag. When the previous distractor stimulus is encountered again, this piece of information is automatically retrieved, thus resulting in NP. Nowadays, researchers agree that inhibitory mechanisms as well as episodic retrieval contribute to NP. Note that beyond inhibition and retrieval, other theories have also been suggested more recently (see Frings, Mast, \& Spence, 2014, for an overview). 
Visual stimuli have been used in the majority of NP studies that have been reported to date. Importantly, though, identitybased NP has also been demonstrated in the auditory modality (e.g., Leboe, Mondor, \& Leboe, 2006; Mayr \& Buchner, 2007), in touch (Frings, Amendt, \& Spence, 2011; Frings, Bader, \& Spence, 2008), and even in olfaction (Olsson, 1999). Furthermore, Buchner, Zabal, and Mayr (2003) induced crossmodal identity-based NP using both auditory and visual stimuli. In their study, the size of the NP effect was the same for all modality combinations. Such a pattern of results might lead to the suggestion that NP does not differ between the senses. However, when processing difficulty has been controlled for, it turns out that tactile NP effects tend to be larger than their visual counterparts (see Frings et al., 2011), thus indicating that identity-based NP yet may not be completely modality-independent. Still, the same theoretical approaches can be used to explain identity-based NP in all sensory modalities.

In contrast to identity-based NP, in which the location of the target and distractor are not critical, in location-based or spatial NP, the position of the target defines how to respond. That is, in this variant of the NP task, responses toward targets are slowed when they are presented from the location where a distractor had previously been presented. This performance decrement represents the spatial NP effect (see, e.g., Tipper, Brehaut, \& Driver, 1990). Spatial NP effects can be explained on the basis of both inhibition and retrieval theories. According to retrieval-based accounts, spatial NP is caused by the automatic retrieval of an episodic memory in which the current target location was ignored (e.g., Neill \& Valdes, 1992). Inhibition theories share the central claim that residual inhibition of the prime distractor causes spatial NP. On the one hand, it has been suggested that spatial NP is due to residual inhibition of the internal representation of the location of the prime distractor (Baylis, Tipper, \& Houghton, 1997; Milliken, Tipper, Houghton, \& Lupiáñez, 2000; Tipper et al., 1990). Note, however, that in the original spatial NP paradigm, once again both the location of the prime distractor and the associated response are involved on ignored repetition trials, but not on control trials. Hence, on the other hand, response inhibition may also contribute to spatial NP (see Buckolz, Goldfarb, \& Khan, 2004). Furthermore, Park and Kanwisher (1994) have suggested that feature mismatch may cause spatial NP. These authors took into account that on ignored repetition trials, in contrast to control trials, stimuli with different determining features are regularly encoded at the same location (i.e., the prime distractor stimulus is presented at the same location as the probe target). That is, according to feature-mismatch accounts, perceptual interference while encoding the probe target is assumed to elicit NP.

Spatial NP has been demonstrated in vision (Chao, 2009; Milliken, Tipper, \& Weaver, 1994), audition (Mayr, Buchner, Möller, \& Hauke, 2011; Mayr, Hauke, \& Buchner, 2009; Mayr, Möller, \& Buchner, 2014; Möller, Mayr, \& Buchner,
2013), and touch (Frings et al., 2014). Interestingly, however, the available research indicates that the processes underlying spatial NP differ between these sensory modalities. Specifically, visuospatial NP effects depend on the repetition of the prime distractor response during the probe display, not on requiring the repetition of the prime distractor location. That is, the inhibition of the prime distractor response has been demonstrated to be the main cause of visuospatial NP (e.g., Buckolz et al., 2004). By contrast, no significant contribution of response inhibition to auditory NP has been observed (Möller et al., 2013). Conversely, a change in the selection criterion (i.e., the prime distractor stimulus is used as the probe target stimulus) has been shown to eliminate spatial NP in audition but not in vision. In other words, auditory spatial NP is eliminated when there is no mismatch between the stimulus features encoded at the repeated location (prime distractor, probe target). That is, feature mismatch is crucial for auditory spatial NP (Mayr et al., 2009; Mayr et al., 2014; Möller et al., 2013), yet it does not impact strongly on visuospatial NP (Milliken et al., 1994; Tipper, Weaver, \& Milliken, 1995). Taken together, visuospatial NP has been shown first to reflect the aftereffects of the inhibition of previously activated responses, and second, is independent of feature mismatch. By contrast, auditory spatial NP is only observed when a new auditory stimulus occurs at the previously ignored location (and not when the prime distractor stimulus itself is repeated at the same location, serving as the target stimulus during the probe display), and thus seems to depend entirely on feature mismatch. Yet, no aftereffects of ignoring are observed in audition when responding to the probe target requires the execution of the prime distractor response without the repetition of the prime distractor location during the probe (reflecting the impact of mere response inhibition on spatial NP).

In the tactile modality, it has recently been shown that spatial $\mathrm{NP}$ is not modulated by feature mismatch. Specifically, Frings et al. (2014) demonstrated that the size of tactile spatial NP effects does not vary as a function of the presence versus absence of feature mismatch at the location from which the prime distractor and the probe target are presented. Remarkably, this pattern of results indicates that the processes contributing to spatial NP differ for audition and touch. Notably, Frings et al.'s (2014) study does not enable one to draw any conclusions as to whether tactile spatial NP is sensitive to locationassociated processes or whether instead the inhibition of the response associated with the prime distractor is what drives spatial NP in touch - as is the case in vision. The aim of the present study was therefore to investigate whether touch behaves like vision in spatial NP. That is, we investigated for the first time the role of response inhibition in tactile spatial NP. To investigate this issue, we developed a new tactile variant of the spatial NP paradigm. Crucially, as in those spatial NP paradigms used by Buckolz et al. (2004), the classical one-to-one mapping between the stimuli and responses was therefore eliminated in the present study (though we did so by different means; see 
below). Specifically, Buckolz et al. (2004) included a freechoice mapping for one location. As a result, on some trials the participant's probe response was a repetition of the prime distractor response without the probe location being a repetition of the prime distractor location. Buckolz et al.'s (2004) results revealed insights concerning the locus of visuospatial NPnamely, that it is largely attributable to response inhibition. To the contrary, in the present study we introduced a variant of spatial NP in which multiple locations were mapped onto the same response (see Buckolz, Edgar, Kajaste, Lok, \& Khan, 2012; and see Guy, Buckolz, \& Khan, 2006, for studies using similar many-to-one location-to-response mappings). In this way, we were also able to disentangle the contributions to spatial NP of (a) mechanisms associated specifically with the prime distractor location (which may either be inhibitory or retrievalbased) and (b) the inhibition of the prime distractor response. If response inhibition represents the main cause of tactile spatial NP, then NP effects should be observed when the probe target occurs at the prime distractor location (location repetition, including response repetition) and also when the probe target occurs at a different location than the prime distractor but is mapped onto the same response as the prime distractor (mere response repetition).

\section{Method}

\section{Participants}

A total of 33 students from the University of Trier took part in this experiment in exchange for either monetary payment or course credit. Their median age was 23 years (ranging from 19 to 37 years). All of the participants had a normal sense of touch and normal or corrected-to-normal vision by self-report, and they were all naïve as to the purpose of the study.

\section{Design}

The experiment consisted of a 3 (Trial Type: location repetition vs. response repetition vs. control) $\times 2$ (Selection Criterion $^{1}$ : change vs. no change) within-participants experimental design. The average reaction time (RT) was the main dependent variable, though error rates were also analyzed.

\footnotetext{
${ }^{1}$ Importantly, the Selection Criterion factor reflects a manipulation of the presence versus absence of feature mismatch (i.e., of perceptual interference during probe encoding) regarding location repetition trials. Yet this does not hold regarding response repetition and control trials. This is because there is no perceptual interference during encoding on the latter types of trials, simply because no location is repeated on these trials. Consequently, there is no feature mismatch on response repetition and control trials.
}

First, performance on location repetition trials was compared to that on control trials. This contrast reflects the standard spatial-NP effect. Note that the comparison of the sizes of this effect between change and no-change trials constitutes a replication of Frings et al. (2014). There is no feature mismatch on change trials, since the identity of the target changes from the prime to the probe display. That is, on location repetition trials on which the selection criterion changes, the same stimulus represents the prime distractor and the probe target and is presented twice from the same location. In contrast, on the no-change trials, the identity of the target remains the same during the prime and probe displays, resulting in feature mismatch on location repetition (but not on control) trials, since in this case different stimuli serve as the prime distractor and probe target, respectively.

Second, crucially, performance on response repetition trials was compared to that on location repetition trials. This contrast represents the pure effect of responding to a location that had previously been occupied by a distractor (i.e., on location repetition trials, the probe includes repetition of the location features of the prime distractor, and it also requires the execution of the response associated with the prime distractor; yet, on response repetition trials, the prime distractor response is required without the prime distractor location being occupied on the probe). Then again, comparing performance on response repetition trials to that on control trials provides a measure of the impact of executing the prior distractor response in the absence of the repetition of other location features.

Given $N=33$, the power to detect medium-sized effects in the binary comparisons of the Trial Type factor (i.e., $d_{z}=0.5$, $\alpha=.05$ ) was $1-\beta=.88$ (G*Power; Faul, Erdfelder, Lang, \& Buchner, 2007).

\section{Apparatus and materials}

The experiment was conducted on a standard PC with a 24-in. CRT screen. E-Prime (Version 2.0) was used to run the experiment and to control the presentation of the vibrotactile stimuli via a serial interface. The vibrotactile stimuli were delivered by means of six tactors (Model C-2, Engineering Acoustic, Inc.) that were driven independently from the PC by an individual standard amplifier (Power Amplifier Module PM40C, t.amp). Each tactor was 1.17 in. in diameter and 0.30 in. thick.

Two vibrotactile stimuli were used in this experiment. The first vibration consisted of a continuous pulse, and the second vibrotactile stimulus consisted of 100-ms pulses separated by 110 -ms pauses. On a given trial of the experimental phase, both stimuli were presented. Each of the stimuli represented the target on some trials and the distractor on others, with the identity of the target being indicated at the beginning of each trial. Throughout the experiment, the stimuli on a given trial were presented repeatedly until a response was detected. 


\section{Procedure}

The participants were tested individually in a soundproof and dimly lit room with all sources of illumination eliminated. They sat $60 \mathrm{~cm}$ from the display, with the head resting on a chinrest. The participants' hands were positioned palms-down in front of their bodies. The tactors were fastened onto the insides of the participant's wrists, elbows, and both upper arms by means of Velcro strips. White noise was presented via headphones in order to mask any potential auditory cues emitted by the operation of the tactors. The participants also put their wrists on armrests to reduce the noise made by the tactors (in the case they were touching the table, for example). On each trial, the participants' task was to respond to the target (and to ignore the distractor) by pressing the key associated with the target location. In the experimental phase, the six possible locations were thus mapped onto four response keys. Targets presented at the left (or right) wrist or forearm required the participant to press the "D" key (or "J" key) with the left (or right) middle finger, whereas targets presented at the left (or right) upper arm required to press the " $\mathrm{C}$ " key (or " $\mathrm{N}$ " key) with the left (or right) index finger instead. Thus, the locations of the appropriate response keys spatially corresponded to the locations of the vibrotactile stimuli (see Fig. 1). Instructions were provided on the screen.

The experimental session comprised four learning phases, in which participants learned the mapping of the stimulus locations to appropriate response keys and familiarized themselves with the task. That is, the task difficulty was increased from one learning phase to the next (e.g., only four possible locations were used during the learning phases at the beginning of the experiment, with the fifth and sixth locations being introduced during the latter learning phases). Then there was one experimental phase in which the participants had to indicate which of the six possible locations the target vibration was presented from on each one of two subsequently presented displays (i.e., the prime and the probe).

In detail, Learning Phase 1 consisted of eight trials, wherein each of the two vibrations was presented once at the right and once at the left wrist and the upper arm (i.e., no stimuli were presented at the forearm during Learning Phases 1-3). The order of trial presentation was randomized. Then, in Learning Phase 2 (consisting of 24 trials), both vibrations were delivered simultaneously to two of the same four locations, in a random order. Specifically, location pairs were randomly selected from a preset list containing all possible combinations of any two out of the four locations. Actually, each combination of two Locations A and B occurred four times in this list-once with Stimulus 1 serving as the target at Location A and Stimulus 2 serving as the distractor at
Location B, once with Stimulus 2 serving as the target at Location A and Stimulus 1 serving as the distractor at Location $\mathrm{B}$, and the same combinations with the locations reversed (i.e., with the target being presented from Location $\mathrm{B}$ and the distractor stimulus from Location A). To indicate which of the vibrations constituted the target on a given trial, a green "1" or a blue "2" was presented at the center of the screen for $500 \mathrm{~ms}$ before the vibrotactile stimulus was presented. Specifically, a green "1" indicated that the participant should respond to the location of the continuous vibrotactile stimulus, whereas a blue " 2 " indicated that the participant should respond to the intermittent vibrotactile stimulus.

In Learning Phase 3 (consisting of 24 trials), each trial comprised a prime display and a probe display (double trials), whereby each display comprised a target vibration as well as a distractor vibration. That is, on each display, both of the vibrotactile stimuli were delivered simultaneously to two of four locations (right/left wrist vs. forearm), and a visual cue (a green "1" or a blue " 2 "), which was presented before the vibrations, indicated the identity of the subsequent target. There were two types of trials in Learning Phase 3. First, there were those trials on which, during the probe display, the target was presented at the location from which the distractor had been presented during the prime display (location repetition). Second, there were those trials on which the probe target and probe distractor were presented at the two remaining locations that had not yet been stimulated during the prime display (control trials). Orthogonal to the manipulation of trial type was a manipulation of the selection criterion (change vs. no change): The identity of the target remained the same during the prime and probe displays on no-change trials (implying that the features of the stimuli presented at the repeated location on location repetition trials [prime distractor/probe target] contained a mismatch), whereas target identity switched between the prime and probe displays on the change trials (resulting in the elimination of feature mismatch on these location repetition trials). That is, during Learning Phase 3, EPrime again randomly selected a combination of locations from a list. Importantly, this list now contained combinations of four locations - namely the prime target location, the prime distractor location, the probe target location, and the probe distractor location (again with no stimulus ever presented on the right/left forearm). These combinations were designed in such a way to represent location repetition trials and control trials, with each stimulus possibly occurring at any of the four locations. Then, on each trial, a combination of four locations was again randomly selected by E-Prime. As later in the experimental phase, the participants initiated the start of each double trial by pressing the space bar.

In Learning Phase 4 (consisting of 12 double trials), six possible locations were used (right/left wrist vs. right/left forearm vs. right/left upper arm). The stimuli presented to the left (or right) forearm required the same response as the stimuli 


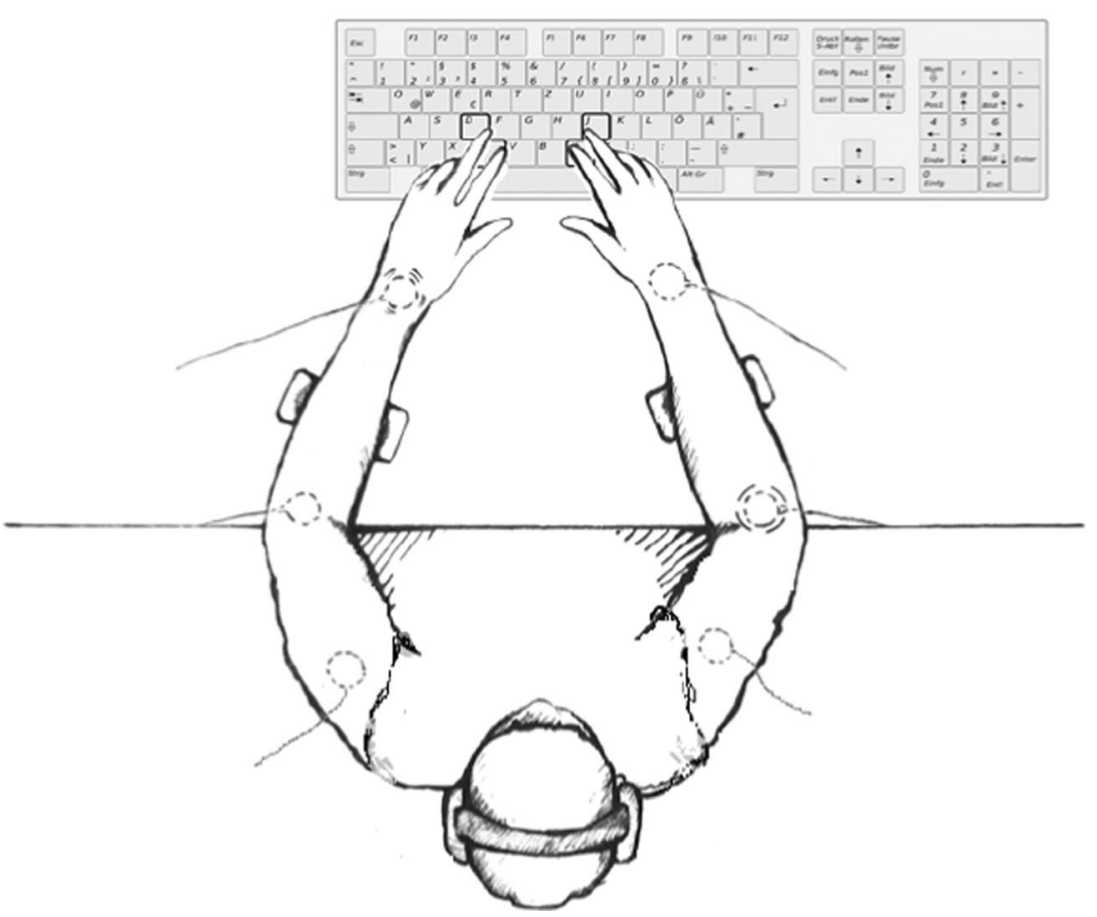

Fig. 1 Schematic depiction of the experimental setup. Adapted from "Tactile Spatial Negative Priming Occurs Without Feature Mismatch," by C. Frings, F. Mast, and C. Spence, 2014, Attention, Perception, \&

delivered to the left (or right) wrist, so that there were four possible responses. That is, there was a one-to-one locationto-response association for the upper-arm locations, and a two-to-one location-to-response association for the remaining locations (right wrist and forearm, left wrist and forearm). As a result, there was one extra trial type, in addition to the location repetition and control trials that had already been introduced (though this was not, of course, explicitly explained to the participant) in Learning Phase 3-namely, response repetition trials (see Fig. 2 for an overview of the possible trial types). On these trials, the targets presented during the probe display and the distractors presented during the prime display were presented from two distinct locations that were mapped onto the same response (e.g., the left forearm and the left wrist). In an exemplary response repetition trial, the distractor was presented to the right forearm during the prime display, and the target was presented to the right wrist during the probe display. In this case, then, the ignored response (i.e., pressing the "J" key) was repeated, but the ignored location was not. Hence, on response repetition trials, the execution of the response that could have been inhibited during the prime display was required during the probe display (as was the case on location
Psychophysics, 76, 2305-2314. Copyright 2014 by the Psychonomic Society

repetition trials). Yet, the stimulus location of the prime distractor was not repeated during the probe display on response repetition trials (this was, hence, only the case on location repetition trials). ${ }^{2}$

Note that on control trials, with six possible locations, none of the probe stimuli were ever presented from the prime target location or the prime distractor location. Rather, the probe locations on control trials had never been stimulated during the prime display. Furthermore, neither the probe target nor the probe distractor was ever presented from the second location mapped onto the same response as the prime target location on control trials. In addition, the probe distractor was never presented from the second location mapped onto the same response as the prime distractor location. Yet, the probe target occurred at one of the locations with a two-to-one

\footnotetext{
${ }^{2}$ Note that on response repetition trials, the probe target was never presented from any of the upper-arm locations (and neither was the prime distractor). That is, in the present paradigm, the effects of response and location repetition cannot be disentangled for these two locations. A design with four two-location-to-one-response mappings would be needed to enable this disentanglement for all locations. This was not necessary for the investigation of our hypothesis.
} 


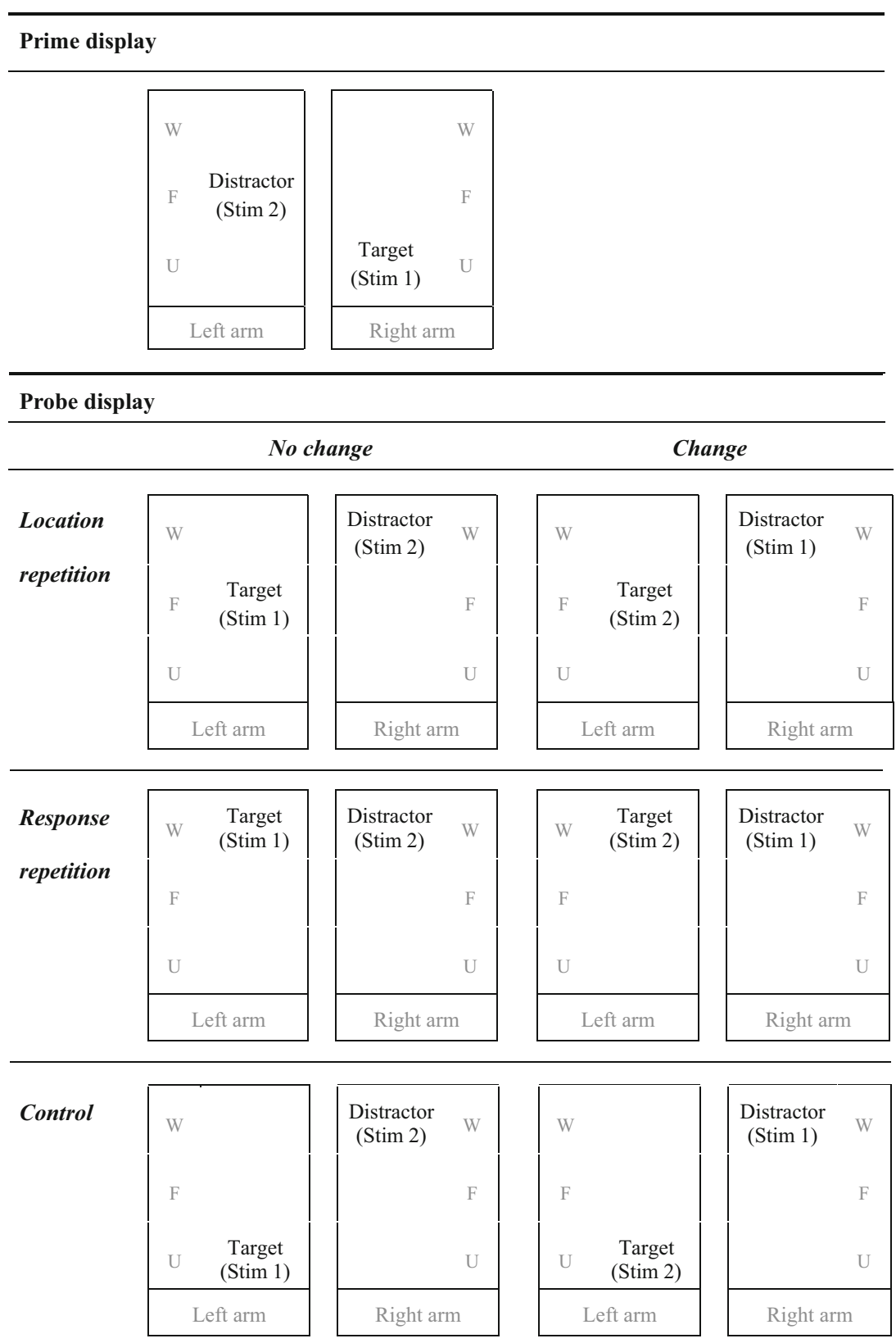

Fig. 2 Examples of the vibrotactile stimuli and locations at which they were delivered for the different conditions of trial type (location repetition vs. response repetition vs. control) and selection criterion (change vs. no change). See the text for further details. $\mathrm{W}=$ wrist, $\mathrm{F}=$ forearm, $\mathrm{U}=$ upper arm

prime and probe target/distractor to these locations during Learning Phase 4, and also during the subsequent experimental phase. Apart from the addition of two locations, Learning Phase 4 constituted a replication of Learning Phase 3. Since, in all of the learning phases, the participants received feedback as to whether or not their response on a given trial had been correct, we were able to ensure that the participants learned the stimulus-response mapping.

When the participants had finished the learning phases, the experimental phase started. Here, the 
participants once again initiated each double trial by pressing the space bar. The visual cue indicating the identity of the target was then presented for $500 \mathrm{~ms}$. Next, after a delay of $100 \mathrm{~ms}$, the vibrotactile prime stimuli were repeatedly delivered to two of the six possible locations until a response had been detected. If the response to the prime target was incorrect, feedback was presented for $200 \mathrm{~ms}$. Then the cue indicating the identity of the probe target was presented $(500 \mathrm{~ms})$. After a response toward the probe stimulus had been detected, feedback was again presented in the case of an incorrect response. For the prime as well as the probe display, a black screen was presented for $200 \mathrm{~ms}$ after the detection of the response, in the case of a correct response. After the probe feedback/black screen, the next double trial started. The experimental phase comprised 80 location repetition trials, 80 response repetition trials, and 80 control trials, giving rise to a total of 240 double trials. Within each trial type, the selection criterion (i.e., reflecting which of the two stimuli is to be selectedthat is, which of the stimuli represents the target) changed from the prime to the probe displays on half of the trials, whereas the selection criterion did not change on the other half of the trials. As a result, half of the location repetition trials contained a feature mismatch (i.e., no-change trials).

\section{Results}

Only probe responses following correct prime responses were included in the analyses of the RT data. Furthermore, RTs had to be longer than $200 \mathrm{~ms}$ and lower than the Tukey (1977) outlier criterion (i.e., three interquartile ranges above the third quartile of each participant's individual RT distribution). Due to these restrictions, $40.5 \%$ of all trials were excluded from the analyses (the average probe error rate was $24.8 \%$ ). The probe mean RTs and probe error rates are highlighted in Table 1. For all of the statistical analyses reported here, a significance level of $\alpha=.05$ was specified.

\section{Reaction times}

A 3 (Trial Type: location repetition vs. response repetition vs. control $) \times 2$ (Selection Criterion: change vs. no change) multivariate analysis of variance (MANOVA) was conducted. The analysis revealed no significant main effect of selection criterion, $F<1$, but a significant main effect of trial type, $F(2,31)$ $=7.64, p=.002, \eta_{\mathrm{p}}{ }^{2}=.33$. No interaction effect emerged, $F(2$, $31)=1.53, p=.233, \eta_{\mathrm{p}}{ }^{2}=.09$. Contrasts probing the main effect of trial type revealed that RTs on location repetition trials ( $M=1,633 \mathrm{~ms}, S D=536 \mathrm{~ms})$ were significantly longer than those on response repetition trials $(M=1,506 \mathrm{~ms}, S D=$ $456 \mathrm{~ms}), p=.002$. This means that responses toward probe targets presented at the location previously occupied by a distractor were more strongly impaired than those responses toward probe targets occurring at a new location but still requiring the prime distractor response (see Fig. 3). Moreover, contrasts revealed no significant difference between RTs on response repetition and control trials $(M=1,490 \mathrm{~ms}, S D=$ $541 \mathrm{~ms}), p=.564$, indicating no impact of executing the response that had previously been associated with the distractor.

Further analyses revealed that responses were prolonged on location repetition as compared to control trials, $F(1,32)=$ $15.12, p<.001, \eta_{\mathrm{p}}^{2}=.32$, reflecting the standard spatial NP effect. Importantly, the standard NP effect was significant on change trials $(M=109 \mathrm{~ms}, S D=199 \mathrm{~ms}), t(32)=3.59, p=$ $.001, d=0.63$, as well as on no-change trials $(M=178 \mathrm{~ms}, S D$ $=284 \mathrm{~ms}), t(32)=3.14, p=.004, d=0.55$. The difference between these conditions was not significant, $F(1,32)=2.54$, $p=.121, \eta_{\mathrm{p}}{ }^{2}=.07$, thus replicating the findings reported by Frings et al. (2014).

\section{Error rates}

For the error rates, responses toward the probe target on those trials with a correct prime response were analyzed. Therefore, the same $3 \times 2$ MANOVA as for the RTs was conducted with regard to the error rates. Once again, the analysis revealed a significant main effect of trial type, $F(2,31)=5.22, p=.011$, $\eta_{\mathrm{p}}{ }^{2}=.25$. Still, neither the main effect of selection criterion,

Table 1 Mean reaction times (RTs) in milliseconds and error rates in percent as a function of trial type (location repetition vs. response repetition vs. control) and selection criterion (change vs. no change)

\begin{tabular}{|c|c|c|c|c|c|c|c|}
\hline & & \multicolumn{6}{|l|}{ Trial Type } \\
\hline & & \multicolumn{2}{|c|}{ Location Repetition } & \multicolumn{2}{|c|}{ Response Repetition } & \multicolumn{2}{|l|}{ Control } \\
\hline & & RTs & Errors & RTs & Errors & RTs & Errors \\
\hline \multirow[t]{2}{*}{ Selection criterion } & Change & $1,643(564)$ & $30.5(20.9)$ & $1,519(475)$ & $24.1(17.5)$ & $1,465(456)$ & $23.3(20.8)$ \\
\hline & No change & $1,624(542)$ & $27.3(22.0)$ & $1,493(476)$ & $24.3(18.9)$ & $1,515(475)$ & $19.5(15.5)$ \\
\hline
\end{tabular}

Only RTs greater than $200 \mathrm{~ms}$ and lower than the individual Tukey (1977) outlier criterion are included, and only trials with correct prime responses are included. Standard deviations are in parentheses 


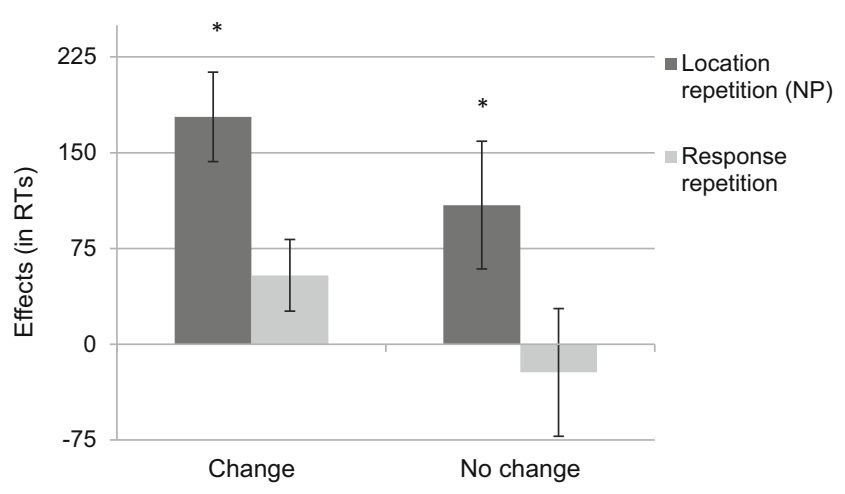

Fig. 3 Graph showing the sizes of the effects (on reaction times) attributable to the repetition of the prime distractor location (location repetition minus control trials), as compared to the effects attributable to the repetition of the prime distractor response (response repetition minus control trials), as a function of selection criterion (change vs. no change). The error bars indicate the standard errors of the means. ${ }^{*} p<.05$

$F(1,32)=3.83, p=.059, \eta_{\mathrm{p}}{ }^{2}=.11$, nor the interaction between trial type and selection criterion, $F(2,31)=1.04, p=$ $.366, \eta_{\mathrm{p}}{ }^{2}=.06$, was significant. Analyzing the main effect of trial type in detail by means of contrasts revealed that the error rate was significantly higher on location repetition trials $(M=$ $28.9 \%, S D=20.8 \%)$ than on response repetition trials $(M=$ $24.2 \%, S D=17.5 \%$ ), $p=.025$ (one-tailed). Moreover, the error rate on response repetition trials did not differ significantly from that seen on control trials $(M=21.4 \%, S D=$ $17.0 \%), p=.106$. Once again, these findings indicate that responding to a probe target is impeded if this target happens to occur at the location previously occupied by a distractor. Yet, there is no impact of the needed execution of the previous distractor response (prime distractor response) in the probe.

Further analyses revealed that the error-based NP effect (error rate on location-repetition trials minus error rate on control trials) was also significant on the change trials $(M=7.2 \%$, $S D=15.0 \%), t(32)=2.75, p=.010, d=0.48$, as well as on the no-change trials $(M=7.8 \%, S D=16.4 \%), t(32)=2.74, p$ $=.010, d=0.48$. Once again, we found no main effect of selection criterion with regard to the magnitude of spatial NP, $F<1$. These findings therefore constitute a replication of the previous results, indicating that, in touch at least, feature mismatch does not modulate spatial NP (Frings et al., 2014).

\section{Discussion}

In the present study, we investigated the locus of spatial NP in touch. Specifically, we investigated whether the inhibition of the prime distractor response is the primary factor underlying tactile spatial NP. Recently, Frings et al. (2014) demonstrated that the size of tactile spatial NP is not modulated by whether or not there is a mismatch between the target and distractor features presented at the repeated location (i.e., the prime distractor and the probe target). This pattern of results is in line with what has previously been obtained in vision (see, e.g., Milliken et al., 1994; Tipper et al., 1995), whereas it contrasts with what has been seen in audition (where spatial NP was evident only when feature mismatch was present; Mayr et al., 2009; Mayr et al., 2014; Möller et al., 2013). Thus, Frings et al. (2014) provided indicative evidence that touch behaves like vision when it comes to spatial NP, insofar as in touch and vision the perceptual interference during probe encoding seems not to be as important for spatial NP. Turning to visuospatial NP, the aftereffect of the repetition of the prime distractor response as a probe target response (without repetition of the prime distractor location) is sufficient to induce NP (Buckolz et al., 2004). Evidence regarding the role of response inhibition in tactile spatial NP has thus far been lacking.

To address this issue, we implemented a spatial NP paradigm, with participants being instructed to indicate the location of the target by pressing the response key associated with it. Importantly, multiple locations were mapped onto the same response in our study (i.e., two out of the four possible responses were correct with regard to two out of the six possible locations each). As a result, for some prime-probe combinations the prime distractor location was repeated as the probe target location (location repetition trials, similar to ignored repetition trials in the standard spatial NP paradigm). Furthermore, other prime-probe combinations featured repetition of the prime distractor response as the probe target response without repetition of the prime distractor location (response repetition trials). As compared to the control trials on which both stimulus locations occupied during the probe display had not been involved during the prime display, RTs were prolonged on location repetition trials, reflecting the standard spatial NP effect. However, the RTs did not differ on response repetition trials (on which the prime distractor location was not repeated), as compared to control trials.

In the present study, one might wonder, on the basis of the relatively high error rates, whether the participants actually learned the location-response mapping. If this were not the case, then the prerequisite activation of the associated response, which is needed for an impact of the response locus to be observed (see Schlaghecken, Rowley, Sembi, Simmons, \& Whitcomb, 2007) would be lacking. We thoroughly analyzed the probe errors to address this possibility. In detail, we argue that a weak location-response mapping would lead to many random errors (i.e., participants just pressing one of the three keys, which would lead to an error). Remarkably, we did not observe a random distribution of the probe error rates. Rather, probe errors were due to distractor interference (the average proportion of probe trials on which the participants erroneously responded by pressing the key associated with the probe distractor was $69.6 \%$ ); as a result, one might argue that participants were able to respond to the location where a particular stimulus occurred, but obviously they had problems separating the target from the distractor. Of course, we cannot 
rule out the possibility that response-location associations were still not strong enough to elicit strong response interference effects, which may explain the weaker effect of response repetitions.

Taken together, the results reported here demonstrate no impact of response inhibition on the magnitude of tactile spatial NP. That is, our experiment shows a robust tactile spatial NP effect that cannot be explained entirely by response-level inhibition, since RTs in the location repetition condition (in which both the location and response associated with the target during the probe display were ignored during the prime display) were significantly longer and error rates were higher than those in the response repetition condition (in which the probe response, but not the probe target location, was ignored during the prime display). As such, the mechanism responsible for the effect seen here must be sensitive to the location and not solely to the response. This interpretation is further supported by the nonsignificant comparison between performance on response repetition trials and control trials (in which neither the probe response nor the probe target location was ignored during the prime display). However, note that this, of course, does not rule out the possibility of greater responselevel involvement if the location-response associations were made stronger. The present results convincingly show that the requirement to execute the prior distractor response is not the primary source of spatial NP in touch. That is, they indicate that touch does not behave like vision with regard to spatial NP. Furthermore, as in Frings et al.'s (2014) study, feature mismatch did not modulate tactile spatial NP in the present study. This has, in contrast, repeatedly been shown to be the case in audition.

Still, our findings do not demonstrate that tactile spatial NP is completely unaffected by response inhibition and feature mismatch. However, they suggest that neither of the processes to which spatial NP has mainly been attributed in vision (i.e., response inhibition) and in audition (i.e., feature mismatch) is sufficient to cause tactile spatial NP on its own. In conclusion, the results of the present study suggest that the processing of spatial distractors is modality-specific. In vision, spatial NP mainly represents response inhibition, whereas in audition, it is largely attributable to the requirement to encode mismatching features at the same location. In touch, however, spatial NP seems to primarily reflect processes associated with the prime distractor location. Note, though, that the present study does not specifically reveal the processes underlying spatial tactile NP. Future research will be needed to investigate, for example, the contribution of location-associated inhibitory processes, on the one hand, and location-associated retrieval processes, on the other.

Importantly, there are still some methodological drawbacks when comparing spatial NP between the modalities of vision, audition, and touch. In the visuospatial variant of the task, the stimulus locations are positioned within a small area on the screen. In the auditory variant, the stimuli are located farther apart, as they originate from loudspeakers placed within the lab (although one might use tiny loudspeakers to make the distance more comparable to that in vision). The distance of the stimuli in the tactile variant may lay in-between, with an average $25-\mathrm{cm}$ distance from one possible stimulus location to the adjacent location on the same arm (and an average of about a $50-\mathrm{cm}$ distance from one possible stimulus location to the corresponding location on the other arm). These differences among the experimental paradigms may partly explain why there is a shift of the weight of the cognitive processes involved in spatial NP among the senses.

Nonetheless, our results do show that the location of tactile stimuli on the body surface may well have a higher impact on information processing than is the case with the spatial restriction of bottom-up visual and auditory processing. Note that the neuropsychological structure furthering visual, auditory, and tactile information processing likely supports just such a claim. For example, the primary somatosensory cortex is organized spatiotopically with regard to the somatotopic structure, as contrasted with the visual cortex (which is also organized spatiotopically, but with regard to external space) and the primary auditory cortex (which is organized tonotopically; see Spence \& Driver, 2004).

On the basis of the behavioral data obtained in this research, it can be concluded that in touch, it is all about location in the case of spatial NP. This opens the door for intriguing questions regarding the processing of spatial distractors across the senses, as this evidence from spatial NP research suggests modality-specific processes.

Author note The research reported in this article was supported by a grant of the German Research Foundation (DFG) to Christian Frings and Charles Spence (Grant No. FR 2133/5-1). We thank Caroline Bermes for drawing Fig. 1.

\section{References}

Baylis, G. C., Tipper, S. P., \& Houghton, G. (1997). Externally cued and internally generated selection: Differences in distractor analysis and inhibition. Journal of Experimental Psychology: Human Perception and Performance, 23, 1617-1630. doi:10.1037/0096-1523.23.6. 1617

Buchner, A., Zabal, A., \& Mayr, S. (2003). Auditory, visual, and crossmodal negative priming. Psychonomic Bulletin \& Review, 10, $917-$ 923.

Buckolz, E., Edgar, C., Kajaste, B., Lok, M., \& Khan, M. (2012). Inhibited prime-trial distractor responses solely produce the visual spatial negative priming effect. Attention, Perception, \& Psychophysics, 74, 1632-1643. doi:10.3758/s13414-012-0366-0

Buckolz, E., Goldfarb, A., \& Khan, M. (2004). The use of a distractorassigned response slows later responding in a location negative priming task. Perception \& Psychophysics, 66, 837-845. doi:10. 3758/BF03194977

Chao, H. F. (2009). Revisiting the role of probe distractors in negative priming: Location negative priming is observed when probe 
distractors are consistently absent. Attention, Perception, \& Psychophysics, 71, 1072-1082. doi:10.3758/APP.71.5.1072

Dalrymple-Alford, E. C., \& Budayr, B. (1966). Examination of some aspects of the Stroop color-word test. Perceptual and Motor Skills, $23,1211-1214$

Faul, F., Erdfelder, E., Lang, A. G., \& Buchner, A. (2007). G*Power 3: A flexible statistical power analysis program for the social, behavioral, and biomedical sciences. Behavior Research Methods, 39, 175-191. doi:10.3758/BF03193146

Fox, E. (1995). Negative priming from ignored distractors in visual selection: A review. Psychonomic Bulletin \& Review, 2, 145-173. doi: 10.3758/BF03210958

Frings, C., Amendt, A., \& Spence, C. (2011). When seeing doesn't matter: Assessing the after-effects of tactile distractor processing in the blind and the sighted. Journal of Experimental Psychology: Human Perception and Performance, 37, 1174-1181. doi:10.1037/ a0022336

Frings, C., Bader, R., \& Spence, C. (2008). Selection in touch: Negative priming with tactile stimuli. Perception \& Psychophysics, 70, 516523. doi:10.3758/PP.70.3.516

Frings, C., Mast, F., \& Spence, C. (2014). Tactile spatial negative priming occurs without feature mismatch. Attention, Perception, \& Psychophysics, 76, 2305-2314. doi:10.3758/s13414-014-0721-4

Frings, C., Schneider, K. K., \& Fox, E. (2015). The negative priming paradigm: An update and implications for selective attention. Psychonomic Bulletin \& Review, 22, 1577-1597. doi:10.3758/ s13423-015-0841-4

Guy, S., Buckolz, E., \& Khan, M. (2006). The locus of location repetition latency effects. Canadian Journal of Experimental Psychology, 60, 307-318. doi:10.1037/cjep2006028

Houghton, G., \& Tipper, S. P. (1994). A model of inhibitory mechanisms in selective attention. In D. Dagenbach \& T. H. Carr (Eds.), Inhibitory processes in attention, memory, and language (pp. 53112). San Diego, CA: Academic Press.

Leboe, J. P., Mondor, T. A., \& Leboe, L. C. (2006). Feature mismatch effects in auditory negative priming: Interference as dependent on salient aspects of prior episodes. Perception \& Psychophysics, 68, 897-910. doi:10.3758/bf03193353

Mayr, S., \& Buchner, A. (2007). Negative priming as a memory phenomenon: A review of 20 years of negative priming research. Journal of Psychology, 215, 35-51. doi:10.1027/0044-3409.215.1.35

Mayr, S., Buchner, A., Möller, M., \& Hauke, R. (2011). Spatial and identity negative priming in audition: Evidence of feature binding in auditory spatial memory. Attention, Perception, \& Psychophysics, 73, 1710-1732. doi:10.3758/s13414-011-0138-2

Mayr, S., Hauke, R., \& Buchner, A. (2009). Auditory location negative priming: A case of feature mismatch. Psychonomic Bulletin \& Review, 16, 845-849. doi:10.3758/PBR.16.5.845

Mayr, S., Möller, M., \& Buchner, A. (2014). Auditory spatial negative priming: What is remembered of irrelevant sounds and their locations? Psychological Research, 78, 423-438. doi:10.1007/s00426-013-0515-7

Milliken, B., Joordens, S., Merikle, P. M., \& Seiffert, A. E. (1998). Selective attention: A reevaluation of the implications of negative priming. Psychological Review, 105, 203-229. doi:10.1037/0033295X.105.2.203

Milliken, B., Tipper, S. P., Houghton, G., \& Lupiáñez, J. (2000). Attending, ignoring, and repetition: On the relation between negative priming and inhibition of return. Perception \& Psychophysics, 62, 1280-1296. doi:10.3758/BF03212130

Milliken, B., Tipper, S. P., \& Weaver, B. (1994). Negative priming in a spatial localization task: Feature mismatching and distractor inhibition. Journal of Experimental Psychology: Human Perception and Performance, 20, 624-646. doi:10.1037/0096-1523.20.3.624

Möller, M., Mayr, S., \& Buchner, A. (2013). Target localization among concurrent sound sources: No evidence for the inhibition of previous distractor responses. Attention, Perception, \& Psychophysics, 75, 132-144. doi:10.3758/s13414-012-0380-2

Neill, W. T., \& Valdes, L. A. (1992). Persistence of negative priming: Steady state or decay? Journal of Experimental Psychology: Learning, Memory, and Cognition, 18, 565-576. doi:10.1037/ 0278-7393.18.3.565

Neill, W. T., Valdes, L. A., Terry, K. M., \& Gorfein, D. S. (1992). Persistence of negative priming: II. Evidence for episodic trace retrieval. Journal of Experimental Psychology: Learning, Memory, and Cognition, 18, 993-1000. doi:10.1037/0278-7393.18.5.993

Olsson, M. J. (1999). Implicit testing of odor memory: Instances of positive and negative repetition priming. Chemical Senses, 24, 347350. doi:10.1093/chemse/24.3.347

Park, J., \& Kanwisher, N. (1994). Negative priming for spatial locations: Identity mismatching, not distractor inhibition. Journal of Experimental Psychology: Human Perception and Performance, 20, 613-623. doi:10.1037/0096-1523.20.3.613

Schlaghecken, F., Rowley, L., Sembi, S., Simmons, S., \& Whitcomb, D. (2007). The negative compatibility effect: A case for self-inhibition. Advances in Cognitive Psychology, 3, 227-240. doi:10.2478/ v10053-008-0027-y

Spence, C., \& Driver, J. (Eds.). (2004). Crossmodal space and crossmodal attention. Oxford, UK: Oxford University Press.

Tipper, S. P. (1985). The negative priming effect: Inhibitory priming by ignored objects. Quarterly Journal of Experimental Psychology, 37A, 571-590. doi:10.1080/14640748508400920

Tipper, S. P. (2001). Does negative priming reflect inhibitory mechanisms? A review and integration of conflicting views. Quarterly Journal of Experimental Psychology, 54A, 321-343. doi:10.1080/ 02724980042000183

Tipper, S. P., Brehaut, J. C., \& Driver, J. (1990). Selection of moving and static objects for the control of spatially directed action. Journal of Experimental Psychology: Human Perception and Performance, 16, 492-504. doi:10.1037/ 0096-1523.16.3.492

Tipper, S. P., Weaver, B., \& Milliken, B. (1995). Spatial negative priming without mismatching: Comment on Park and Kanwisher (1994). Journal of Experimental Psychology: Human Perception and Performance, 21, 1220-1229. doi:10.1037/0096-1523.21.5.1220

Tukey, J. W. (1977). Exploratory data analysis. Reading, MA: Addison-Wesley. 